\title{
Steady State and Dynamics of Driven Diffusive Systems with Quenched Disorder
}

\author{
Goutam Tripathy and Mustansir Barma \\ Tata Institute of Fundamental Research \\ Homi Bhabha Road, Mumbai 400 005, India
}

\begin{abstract}
We study the effect of quenched disorder on nonequilibrium systems of interacting particles, specifically, driven diffusive lattice gases with spatially disordered jump rates. The exact steadystate measure is found for a class of models evolving by drop-push dynamics, allowing several physical quantities to be calculated. Dynamical correlations are studied numerically in one dimension. We conjecture that the relevance of quenched disorder depends crucially upon the speed of the kinematic waves in the system. Time-dependent correlation functions, which monitor the dissipation of kinematic waves, behave as in pure system if the wave speed is non-zero. When the wave speed vanishes, e.g. for the disordered exclusion process close to half filling, disorder is strongly relevant and induces separation of phases with different macroscopic densities. In this case the exponent characterizing the dynamical correlation function changes.
\end{abstract}

Pacs numbers: 05.60.+w, 05.40.+j, 05.50.+q, 05.70.Ln

What is the effect of quenched disorder on driven, nonequilibrium systems? This question is important in a number of physical situations involving flow in random media [1]. Theoretically, our understanding of these phenomena is based largely on numerical simulations and on the analysis of continuum equations for coarse-grained variables. In this Letter we obtain the exact steady state and static correlation function for a class of models of driven, interacting particles on a lattice - the drop-push process - with quenched site disorder in the hopping rates. Further, we study the time-dependence of hydrodynamic fluctuations for this system in one dimension, and also for the disordered asymmetric exclusion process. We find that the behaviour of a system with current $J_{0}$ and density $\rho$ is largely determined by $c_{0}=\partial J_{0} / \partial \rho$. If the density is uniform on a macroscopic scale, $c_{0}$ is the mean speed of kinematic waves which transport density fluctuations through the system [2]. If $c_{0}$ is nonzero, we conjecture that quenched disorder does not affect the asymptotic behaviour of the decay of fluctuations, and support this with extensive numerical results. By contrast, we find that vanishing $c_{0}$ can signal the onset of disorder-induced phase separation with coexisting macroscopic regions of different density, in which case the dynamical behaviour is different.

In a coarse-grained description of a 1- $d$ disordered current-carrying system, the nonuniform steady state density profile is described by a function $\rho_{0}(x)$. The evolution of density fluctuations $\tilde{\rho} \equiv \rho(x, t)-\rho_{0}(x)$ is described phenomenologically by a stochastic evolution equation with spatially random coefficients:

$$
\partial_{t} \tilde{\rho}=\partial_{x}\left[D(x) \partial_{x} \tilde{\rho}-c(x) \tilde{\rho}-\lambda(x) \tilde{\rho}^{2} \ldots-\eta(x, t)\right] .
$$

This follows from the continuity equation $\partial_{t} \tilde{\rho}(x, t)+$ $\partial_{x} J(x, t)=0$ on writing the current as $J(x, t)=$ $J_{s y s}(x, t)-D(x) \partial_{x} \tilde{\rho}+\eta(x, t)$, where $D(x)$ is the spacedependent diffusion constant and $\eta$ is white noise; the systematic part of the current $J_{\text {sys }}$ is expanded as $J_{0}+$ $c(x) \tilde{\rho}+\lambda(x) \tilde{\rho}^{2} \ldots$. The most relevant source of disorder in (11) is the term with coefficient $c(x)$ which represents the space-varying local speed of the kinematic wave of density fluctuations. The problem is equivalent to that of a moving interface in the presence of a certain type of columnar disorder; the interface height $h(x, t)$ is related to $\tilde{\rho}$ by $\tilde{\rho}=\partial_{x} h$. Equation (1) then becomes

$$
\partial_{t} h=D(x) \partial_{x x} h-c(x) \partial_{x} h-\lambda(x)\left(\partial_{x} h\right)^{2} \ldots-\eta(x, t) .
$$

The random coefficients $c(x), \lambda(x)$ now represent columnar disorder in the $2-d h-x$ space. It is important to understand how disorder affects scaling properties and to identify factors responsible for different universality classes [3]. A crucial difference between (2) and the model studied by Krug [ [4] is the absence of an additive quenched noise $\epsilon(x)$ which models a frozen random contribution to interface mobility. Such a term, which strongly influences static and dynamic properties, is necessarily absent in (2) as a consequence of the spatial constancy of $\left\langle J_{\text {sys }}\right\rangle$ implied by particle conservation. Further, our model is distinct from depinning- threshold interface models with quenched point disorder [3]. We show that the roughness exponent $\alpha=1 / 2$ in contrast to [3] and [4]. The decay of the kinematic wave in time leads to a power-law $\sim t^{2 \beta}$ growth of the correlation function

$$
S^{2}(t) \equiv\left\langle\left[h\left(x+c_{0} t, t\right)-h(x, 0)-J_{0} t\right]^{2}\right\rangle .
$$

We conjecture that as long as $c_{0}$ is nonzero, leading power law behaviours are the same as in the pure system with no disorder $\left(\beta=\frac{1}{3}\right)[7,8]$. This is supported by Monte Carlo simulation results for the disordered drop-push process, and for the disordered exclusion process in the regime $|\rho-1 / 2|>\Delta$ with $\Delta \neq 0$. For $|\rho-1 / 2|<\Delta$ we present evidence that $J_{0}$ is independent of $\rho$. In this regime, $c_{0}$ vanishes and shocks separate macroscopic regions of different mean densities.

The disordered drop-push process (DDPP) is a model of driven transport of carriers trapped in local regions of space, with each move involving a cascade of transfers through filled traps [5.6]. In 1- $d$, on every site $i$ of an $L$-site ring is a well which can accommodate at most $l_{i}$ 


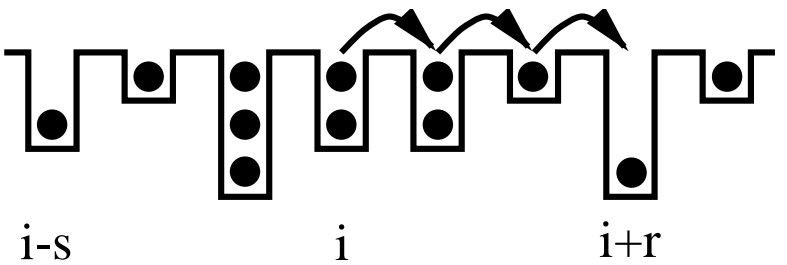

Fig. 1 Typical DDPP configuration and move.

particles (Fig. 1). Each well depth $l_{i}$ is chosen independently from a probability distribution $P\left(l_{i}\right)$. The configuration of the system is specified by the set of particle occupation numbers $\left\{n_{i}\right\}$ of all the wells. The dynamics is stochastic, and in a small time interval $d t$, there is a probability $\epsilon\left(n_{i} \mid l_{i}\right) d t$ that a particle from well $i$ hops out, and drops into well $i+1$. If well $i+1$ is already full, a particle from this well now gets pushed further right, and so on (Fig. 1). The cascade of adjacent-site jumps terminates once a particle drops into a well (say $i+r$ ) which was not completely full earlier. This elementary move thus changes configuration $\mathcal{C} \equiv\left\{\ldots . . n_{i} l_{i+1} \quad l_{i+2} \quad \ldots l_{i+r-1} n_{i+r} \ldots.\right\}$ to $\mathcal{C}^{\prime} \equiv\{\ldots$ $\left.n_{i}-1 l_{i+1} l_{i+2} \quad \ldots l_{i+r-1} n_{i+r}+1 \ldots.\right\}$. The jump rates $\epsilon\left(n_{i} \mid l_{i}\right)$ are pre-specified, and depend both on the depth of the well and the occupation. The rates and well depths are quenched random variables.

The probability for the system to be in configuration $\mathcal{C}$ satisfies the standard master equation [9] with the transition probabilities $W\left(\mathcal{C} \rightarrow \mathcal{C}^{\prime}\right)$ identified with $\epsilon$ 's above. In the steady state, the total flux out of $\mathcal{C}$ equals the total incoming flux. This is ensured if for every $\mathcal{C}^{\prime}$ obtained from $\mathcal{C}$ by an elementary transition there is a unique configuration $\mathcal{C}^{\prime \prime}$ such that in the steady state

$$
W\left(\mathcal{C} \rightarrow \mathcal{C}^{\prime}\right) \mu(\mathcal{C})=W\left(\mathcal{C}^{\prime \prime} \rightarrow \mathcal{C}\right) \mu\left(\mathcal{C}^{\prime \prime}\right)
$$

Here $\mu(\mathcal{C})$ is the invariant measure and (4) is the condition of pairwise balance [6]. We define weights for single-site occupations by $u_{i}(0)=1$ and $u_{i}\left(n_{i}\right)=$ $\tau_{i}(1) \tau_{i}(2) \cdots \tau_{i}\left(n_{i}\right)$ if $0<n_{i} \leq l_{i}$. with $\tau\left(n_{i}\right) \equiv$ $\epsilon_{0} / \epsilon\left(n_{i} \mid l_{i}\right)$, where $\epsilon_{0}$ is a microscopic rate. The measure for configuration $\mathcal{C} \equiv\left\{n_{i}\right\}$ has the product form

$$
\mu(\mathcal{C})=\prod_{i=1}^{L} u_{i}\left(n_{i}\right)
$$

To show that this satisfies (4), we construct the configuration $\mathcal{C}^{\prime \prime}$ corresponding to a given $\mathcal{C}$ and $\mathcal{C}^{\prime}$ as follows. Suppose the transition $\mathcal{C} \rightarrow \mathcal{C}^{\prime}$ involves hopping a particle at well $i$ to well $i+r$ with all wells in between full. Also suppose well $i-s$ is not full but all wells between $i-s$ and $i$ are full (Fig. 1). Configuration $\mathcal{C}^{\prime \prime}$ is identical to $\mathcal{C}$ at all sites except at the sites $i-s$ and $i$, at which $n_{i-s}^{\prime \prime}=n_{i-s}+1, n_{i}^{\prime \prime}=n_{i}-1$. Then (4) is satisfied, in view of the measure (5). Since the dynamics is ergodic the invariant measure (5) is unique for a fixed number of particles [9]. This generalizes the result obtained earlier for the non-disordered case [6], and is the first instance of an exact determination of the steady state of a spatially disordered nonequilibrium system of interacting particles [10.

The product measure form (5) allows us to calculate several physical quantities: the site densities, heightheight correlation function, current $J_{0}$, and wave speed $c_{0}$. In the limit of large $L$, it is convenient to introduce the generating function $Z_{i}=\sum_{n_{i}=0}^{l_{i}} u_{i}\left(n_{i}\right) z^{n_{i}}$ where $z$ is the fugacity. The probability of occurrence of configuration $\mathcal{C}$ is $\mathcal{P}(\mathcal{C})=\mu(\mathcal{C}) z^{N_{P}} / \prod_{i} Z_{i}$, where $N_{P}$ is the number of particles in the configuration. The steady state is characterized by uniform $z$, but inhomogeneous site densities $\left\langle n_{i}\right\rangle=z \partial \ln Z_{i} / \partial z$. The heightheight correlation function $\Gamma^{2}(r) \equiv\left\langle\left(h_{i+r}-h_{i}\right)^{2}\right\rangle$ becomes $\sum_{j=i+1}^{i+r}\left(\left\langle n_{j}^{2}\right\rangle-\left\langle n_{j}\right\rangle^{2}\right)$ and can be evaluated. Disorder averaging gives $\Gamma(r) \sim r^{1 / 2}$, implying $\alpha=1 / 2$.

To find the steady state current $J_{0}$, note that the current $J_{i, i+1}$ across bond $(i, i+1)$ comes from jumps which either $(a)$ originate from sites to the left of site $i$ (with in-between wells full), or $(b)$ originate from site $i$ itself (a contribution $j_{i}$ ). Class $(a)$ events evidently also contribute to the current across bond $(i-1, i)$. Of all events that contribute to $J_{i-1, i}$, class $(a)$ is that subset of events in which site $i$ is fully occupied. Since the probability of a jump between $i-1$ and $i$ is independent of the probability of occupation of site $i$, we have $J_{i, i+1}=J_{i-1, i} p_{i}\left(l_{i}\right)+j_{i}$ where $j_{i}=\sum_{n_{i}=1}^{l_{i}} \epsilon\left(n_{i} \mid l_{i}\right) p_{i}\left(n_{i}\right)=\epsilon_{0} z\left(1-p_{i}\left(l_{i}\right)\right)$ and $p_{i}\left(n_{i}\right)=u_{i}\left(n_{i}\right) z^{n_{i}} / Z_{i}$. Noting that $J_{i-1, i}=J_{i, i+1}=J_{0}$, we find $J_{0}=\epsilon_{0} z$. Since both $J_{0}$ and $\rho$ are known in terms of $z$, the macroscopic speed $c_{0}=\partial J_{0} / \partial \rho$ of the kinematic wave can be determined [2].

Interestingly, the steady state measure and current can also be found in $d>1$, for models in which the ratio of hopping rates in different directions is independent of site and configuration, and the cascade of adjacent-site overflows in a single move is in the same direction 11].

The other model we investigate is the disordered fully asymmetric simple exclusion process (DASEP) on a 1-d ring. In this model, each site can hold 0 or 1 particle. A particle hop is attempted to the nearest-neighbour site on the right, and is completed only if the site in question is vacant. Attempt rates are associated with bonds and are disordered, with magnitudes varying from bond to bond, chosen from a binary distribution. Unlike in the DDPP, there is no analytical characterization of the steady state even with a single inhomogeneous bond. A numerical study has shown that if $\rho$ is close to $1 / 2$, a single weak bond acts as a blockage and produces unequal densities over macroscopic length scales on either side of it, and a shock where the density profiles meet, far from the blockage [12]. We have studied the disordered case with an extensive number of weak bonds by Monte Carlo simulation and found that the steady state depends strongly on the filling. The current $J_{0}$ varies smoothly with $\rho$ provided $|\rho-1 / 2|>\Delta$ (Regime A), whereas $J_{0}$ has a single value if $|\rho-1 / 2|<\Delta$ (Regime B) (Fig. 2a). The value of $\Delta$ depends on the ratio $r$ of rates of the 

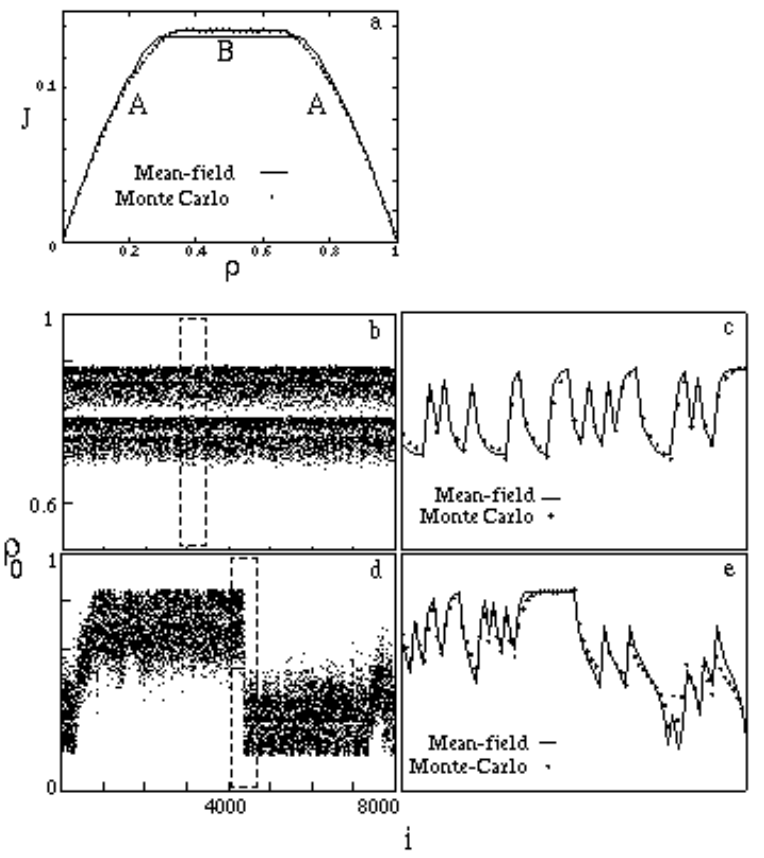

Fig. 2 DASEP steady states (a) $J$ vs $\rho$ for $r=f=0.5$ (b) density profile for $\rho=0.80$ (c) blowup of box in (b) (d) profile for $\rho=0.5$ (e) blowup of box in (d).

weak and strong bonds and on the fraction $f$ of weak bonds, and is $\simeq 0.16$ for $r=f=0.5$. In Regime A, the density profile consists of a large number of shocks with a mean inter-shock spacing of a few lattice spacings (Fig. 2c); the number of shocks scales with the system size. On length scales large compared to the inter-shock spacing, the density is roughly uniform. A semi-quantitative picture of the steady state can be obtained using a meanfield approximation, writing the current between sites $i$ and $i+1$ as $J=\epsilon_{i, i+1} \rho_{0}(i)\left(1-\rho_{0}(i+1)\right)$ where the rate $\epsilon_{i, i+1}$ is $\epsilon_{0}\left(\epsilon_{0} / 2\right)$ for a strong (weak) bond. As $J$ is the same in every bond, the densities $\left\{\rho_{0}(i)\right\}$ satisfy a set of coupled, nonlinear equations, which can be iterated to convergence. The result is shown in Fig. 2c. The mean-field approximation evidently obtains locations of shocks fairly well, but not shapes of individual shocks. In Regime B, Monte Carlo results and mean-field calculations show that the density is nonuniform on a macroscopic scale (Fig. 2d), besides showing shock structure on the scale of a few lattice spacings (Fig. 2e). In this regime, the occurrence of long stretches of successive weak bonds, coupled with the requirement of spatial uniformity of current, results in phase separation into high and low density phases, qualitatively as in the single defect case [12]. In both regimes, numerical results show $\Gamma(r) \sim r^{\alpha}$ with $\alpha \simeq 0.5$

Turning now to the dynamical behaviour of fluctuations in the steady state, we first consider systems which have a uniform density on macroscopic scales. The dynamics of such 1- $d$ systems is dominated by kinematic waves which transport density fluctuations through the system at a mean speed $c_{0}$. Owing to quenched disorder, the local speed of the wave varies from one location to another (Fig. 3) and the question arises how wave dissipation, and thus $S(t)$, is affected. In the pure system, the evolution equation (1) has $x$-independent coefficients and the long-time growth of $S(t)$ is described by $\beta=1 / 3$ [7, 8 . In the disordered system, let us regard the coarse-grained medium as made of successive disordered patches, each independent of the other, and ask for the behaviour of a large-scale density fluctuation as it passes through the succession of patches. If $c_{0}$ is nonzero, the probability of a density fluctuation in an infinite system returning to the same disorder patch dies down rapidly at long times, so it is a good approximation to regard the effect of disorder as essentially uncorrelated in time. Further, since the speed $c(x)$ is a spatially random function, the use of the averaged value $c_{0}$ in (3) induces a noise of amplitude $t^{1 / 2}$ in the location of the density fluctuation. Since fluctuations in $h$ scale as $x^{1 / 2}$, the effect $\left(\sim t^{1 / 2}\right)$ on $S^{2}$ is subleading. Thus we conjecture that if $c_{0} \neq 0$, the long time behaviour of $S^{2}(t)$ is $\sim t^{2 / 3}$, the same as for the pure system. Our argument differs from that used earlier for the effect of point disorder on unpinned, moving interfaces [13], as our case corresponds to columnar disorder in the interface language. The irrelevance of randomness in $c(x)$ is consistent with straightforward power counting in (2).

In our numerical determination of $S(t)$ from simulations of the two types of lattice models, we defined $h(i, t)$ as $\sum_{i_{0}(t)}^{i} \tilde{\rho}(k, t)$ where $i_{0}(t)$ is the position of a specific particle. We averaged results for $S(t)$ over 40 time evolutions for a fixed realization of disorder, and then over several realizations. For drop-push dynamics, we considered a model with only two types of wells $A$ and $B$, distributed randomly. Each well can hold at most one particle, but the jump rates out of the two types of wells are different, say $\epsilon_{A}$ and $\epsilon_{B}$. We used $\epsilon_{A} / \epsilon_{B}=0.5$, and a fraction $f=0.5$ of low rates. Since the placement of the wells is random, the essential feature of quenched disorder is still present. Since (5) still holds, we start with an initial configuration of particles consistent with this product measure. The analysis is aided by the fact that

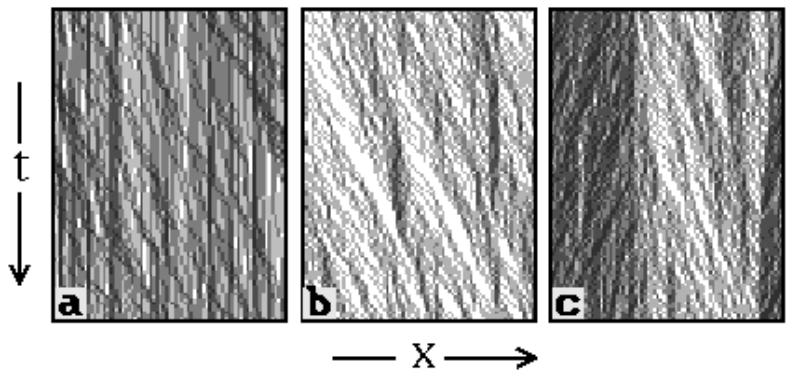

Fig. 3 Typical time evolutions (a) DDPP (b) DASEP, $\rho=0.25$ (c) DASEP, $\rho=0.5$. Darker regions are particlerich. The tilted streaks are kinematic waves. 


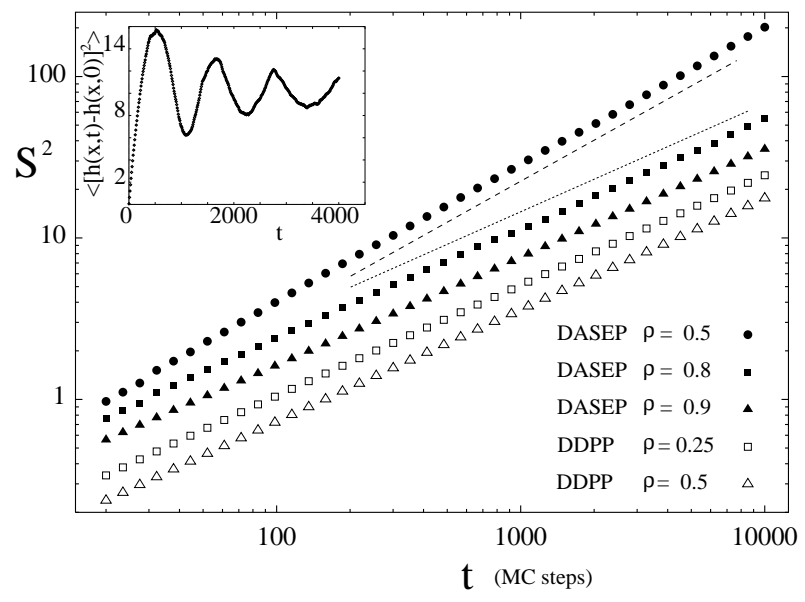

Fig. $4 S^{2}(t)$ for the DDPP and the DASEP for different densities. Individual data sets have been shifted for clarity. The straight lines have slopes 0.84 and 0.67 . Inset: The return time of the kinematic waves is given by the period of oscillations of the autocorrelation function.

$J_{0}$ and $c_{0}$ are known explicitly. In the case of the DASEP, we chose $r=f=0.5$ and used a system of size 8000, allowing it to relax for $\simeq 60,000$ Monte Carlo steps to achieve steady state. Further, $J_{0}$ and $c_{0}$ were estimated in two stages: First, a rough estimate of $J_{0}$ was obtained by direct measurement, while $c_{0}$ (in Regime A) was estimated from the return time of the kinematic wave in a smaller system (Fig. 4 inset). Then a more accurate estimate was obtained by minimizing $S$ in a large system with respect to $J_{0}$ and $c_{0}$. Details of the minimization procedure will be given elsewhere [11]. Fig. 4 shows data for two different densities for the DDPP and also Regime A of the DASEP, all corresponding to nonzero $c_{0}$. In all these cases, the data is consistent with $S^{2} \sim t^{2 / 3}$, as for the pure system, supporting our conjecture. Further, we performed a direct check of the $\sim t^{1 / 2}$ growth of disorder-induced noise in locations of density fluctuations, discussed in the previous paragraph. Such noise should alter the growth law for the sliding-tag correlation function 114 (which monitors fluctuations of taggedparticle locations) from $\sim t^{1 / 3}$ to $\sim t^{1 / 2}-$ a change we confirmed by simulation of the DDPP [11].

In Regime B of the DASEP, $S(t)$ shows stronger fluctuations than in Regime A, from one realization of disorder to another. On averaging over 10 samples, we find $S(t)$ grows as $t^{\beta}$ with $\beta=0.42 \pm 0.02$ (Fig. 4). It is possible that the more rapid growth of $S$ in this case arises from oppositely moving kinematic waves in different macroscopic regions (Fig. 3c).

We conclude with a recapitulation of our principal results. For the DDPP, the steady state has a product measure form, and the current can be determined. For the DASEP, the steady state density profile shows many shocks and is quite well described by a mean-field approximation. Our conjecture, that disorder does not affect the dynamical universality class if the kinematic wave speed $c_{0}$ is nonzero, is borne out by simulation studies of the DDPP and the DASEP in Regime A. A vanishing $c_{0}$ can indicate phase coexistence with different densities in different macroscopic regions, as in Regime B of the DASEP; the dynamical behaviour is different in this case.

We thank R. E. Amritkar, D. Dhar, S. Krishnamurthy and G. I. Menon for useful comments.

[1] O. Narayan and D.S. Fisher, Phys. Rev. B 49, 9469 (1994)

[2] M. J. Lighthill and G. B. Whitham, Proc. R. Soc. London, Ser. A229, 281 (1955); 229, 317 (1955).

[3] L. A. N. Amaral, A. -L. Barabasi and H. E. Stanley, Phys. Rev. Lett. 73, 62 (1994).

[4] J. Krug, Phys. Rev. Lett. 75, 1795 (1995).

[5] M. Barma and R. Ramaswamy in Non-linearity And Breakdown in Soft Condensed Matter, edited by K. K. Bardhan, B. K. Chakrabarti and A. Hansen (Springer, Berlin, 1993), p.309.

[6] G. Schütz, R. Ramaswamy and M. Barma, J. Phys. A29, 837 (1996).

[7] H. van Beijeren, R. Kutner and H. Spohn, Phys. Rev. Lett. 54, 2026(1986).

[8] M. Kardar, G. Parisi and Y. -C. Zhang, Phys. Rev. Lett. 62, 891986.

[9] N. G. van Kampen, Stochastic Processes in Physics and Chemistry, (North Holland, Amsterdam, 1981).

[10] However, models in which quenched random jump rates are associated with particles have been considered in M. R. Evans, Europhys. Lett. 36, 13 (1996) and J. Krug and P. A. Ferrari, J. Phys. A29 L465 (1996).

[11] G. Tripathy and M. Barma, unpublished

[12] S.A. Janowsky and J.L. Lebowitz, Phys. Rev. A45, 618 (1992); G. Schütz, J. Stat. Phys. 75, 471 (1993).

[13] G. Parisi, Europhys. Lett. 17, 673 (1992).

[14] S.N. Majumdar and M.Barma, Phys. Rev. B44, 5306 (1991); Physica A177, 366 (1991). 